\title{
Cryptococcus spp. EM EXCRETAS DE POMBOS (Columba livia) DE ÁREAS PÚBLICAS DE LAGES, SANTA CATARINA
}

\begin{abstract}
MENEZES, Thaianna ${ }^{1}$;
SCAIN, Gustavo ${ }^{2}$;

QUADROS, Rosiléia Marinho3;

MILETTI, Luiz Claudio ${ }^{4}$;

SOUZA, Alexandre Lemos ${ }^{5}$;

MIGUEL, Rafael de Lima ${ }^{6}$;

MARQUES, Sandra Márcia Tietz ${ }^{7}$.

${ }^{1}$ Biomédica, Universidade do Planalto Catarinense - UNIPLAC, ${ }^{2}$ Biólogo - UNIPLAC, ${ }^{3}$ Bióloga, Médica Veterinária, Laboratório de Parasitologia e Zoologia - UNIPLAC, ${ }^{4}$ Bioquímico, Laboratório de Hemoparasitos e Vetores do Centro de Ciências Agroveterinárias - CAV/UDESC, ${ }^{5}$ Biomédico, mestrando em Biotecnologia - UFSC, ${ }^{6}$ Biólogo, Bioquímico, Prof., Laboratório de Microbiologia - UNIPLAC, ${ }^{7}$ Médica Veterinária, Pesquisadora do Departamento de Patologia Clínica Veterinária - UFRGS.
\end{abstract}

\section{RESUMO}

\begin{abstract}
A criptococose é uma infecção fúngica oportunista comum, causada por leveduras capsuladas do gênero Cryptococcus, principalmente pelas espécies $C$. neoformans e $C$. gattii. O fungo é adquirido pela inalação de propágulos de origem ambiental ou mais raramente através de transplantes de órgãos em indivíduos imunocomprometidos. Os pombos domésticos (Columba livia) estão relacionados com a infecção, principalmente como reservatórios naturais do fungo, fato relevante devido a grande concentração destes animais em ambientes públicos, sobretudo em locais de grande circulação de pessoas. Para o isolamento e diagnóstico de Cryptococcus spp., foram analisadas 195 amostras de excretas de pombos de seis praças localizadas na região central da cidade de Lages, SC. Das amostras analisadas, 7,69\% (15) foram positivas para a levedura capsulada. Como as amostras positivas tiveram origem em locais com maior concentração de pombos e fluxo de pessoas, sugere-se a criação de campanhas educativas visando a conscientização da população sobre o risco de contrair a criptococose e a importância de não alimentar os pombos em áreas de lazer, a principal estratégia no controle populacional dos pombos.
\end{abstract}

Palavras-chave: Cryptococcus neoformans. Levedura. Pombos. Zoonose. Praça Pública. 


\section{INTRODUÇÃO}

Fungos do gênero Cryptococcus são compostos por 39 espécies, sendo $C$. neoformans o mais prevalente. É um fungo leveduriforme encapsulado da forma assexuada do basidiomiceto Filobasidiella neoformans (REOLON et al., 2004). O sistema nervoso central (SNC) e o trato respiratório são os órgãos mais acometidos em infecções por C. neoformans e C. gatti; outros órgãos também podem ser infectados como pele, próstata, olhos, ossos, trato urinário e sangue (PASQUALOTTO et al., 2004). A disseminação do agente pode ocorrer em múltiplos órgãos causando infecções em indivíduos imunocomprometidos, sobretudo os infectados pelo vírus da imunodeficiência humana (HIV) (SEVERO et al., 2009). A mortalidade por criptococose é estimada em $10 \%$ nos países desenvolvidos, chegando a $43 \%$ nos países em desenvolvimento (KON, 2008).

O agente, através de suas características morfo-tintoriais, apresenta-se como leveduras globosas ou ovaladas, com brotamento único ou múltiplo, envolvidas por uma espessa cápsula de mucopolissacarídeos, com crescimento colonial de coloração branca a creme, brilhante, de textura mucoide e margem lisa (KWON-CHUNG; BENNETT, 1984; LIN; HEITMAN, 2006). São descritos os sorotipos A, B, C, D e AD (IKEDA et al., 1982; MEYER et al., 2010) e, atualmente, com a classificação através da utilização de minissatélites, os sorotipos foram agrupados em subgrupos: VNI e VNII (variedade grubii - sorotipo A), VNIII (sorotipo AD), VNIV (var. neoformans - sorotipo D) (MEYER et al., 2010) e VGI, VGII, VGIII e VGIV (C. gattii), classificado de acordo com a sequência de multilocus (MLST) e restrição de fragmentos polimórficos (AFLP) (ENGELTHALER et al., 2014). O diagnóstico laboratorial de rotina baseia-se principalmente na visualização das leveduras capsuladas coradas com tinta da China sob microscopia óptica, tanto no isolamento direto como em cultura com ágar Sabouraud ou ágar Níger (FILIÚ et al., 2002).

C. neoformans apresenta ampla distribuição geográfica, ocorre em diversos substratos orgânicos, está frequentemente associado ao habitat de aves, excretas secas, ricas em fontes de nitrogênio, como ureia e creatinina. Condições favoráveis ao crescimento abundante desta levedura formam microfocos, sobretudo em centros urbanos relacionados à presença de pombos (GRANADOS; CASTAÑEDA, 2005; KOBAYASHI et al., 2005; MACHADO 
et al., 1993; QUEIROZ et al., 2008). Os ambientes com grande concentração de excretas favorecem a disseminação da levedura, já que a infecção por $C$. neoformans é adquirida através da inalação de propágulos de origem ambiental por leveduras desidratadas que facilmente se dispersam pelo ar (FILIÚ et al., 2002).

Devido à alta concentração de C. neoformans em excretas de pombos, aliada a alta densidade destas aves em áreas urbanas e a severidade da criptococose humana, existe um crescente interesse no estudo da relação entre as aves, suas excretas e a doença em humanos (HUFFNAGLE, 1999).

Este trabalho objetivou identificar a presença de Cryptococcus spp. em excretas de pombos coletadas de praças da região central da cidade de Lages, Santa Catarina, durante os períodos de inverno de 2011 e 2012.

\section{MATERIAL E MÉTODOS}

A cidade de Lages está localizada na região serrana do estado de Santa Catarina; o clima é subtropical e a temperatura média é de $14,3^{\circ} \mathrm{C}$. A cidade conta com 64 praças entre centrais e periféricas. Para a amostragem foram selecionadas seis praças de um total de dez praças centrais da cidade de Lages, conforme a Figura 1. Para a inclusão das praças foram utilizados o critério de fluxo de pessoas e maior concentração de pombos. 


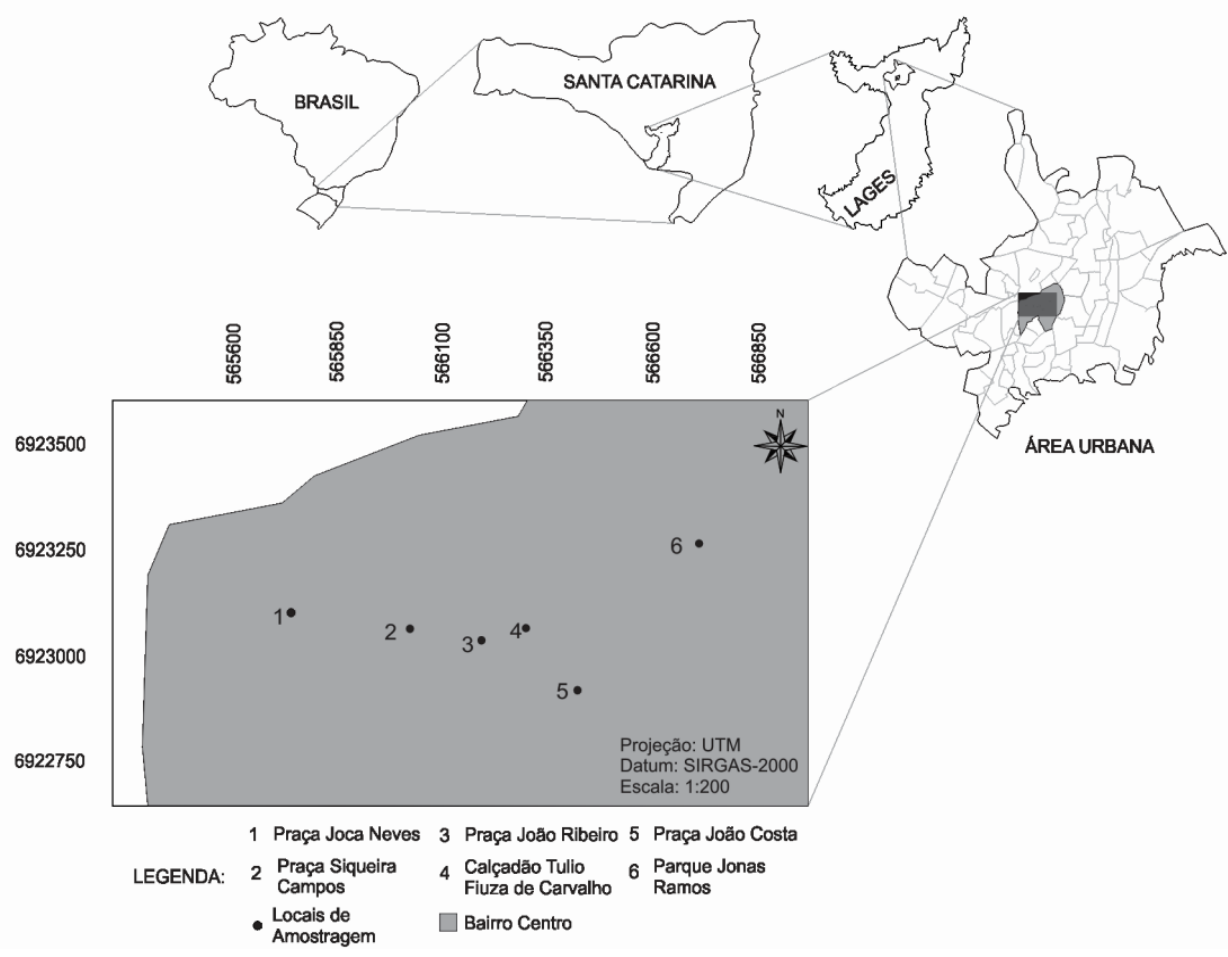

Figura 1 - Representação das seis praças localizadas na área central da cidade de Lages, SC, e distribuídas conforme coordenadas geográficas.

As coletas ocorreram nos meses de junho a setembro nos anos de 2011 e 2012 . Foram realizadas medições nas praças com a finalidade de calcular a área e posterior divisão de quadrantes para possibilitar as coletas das excretas de pombos. Quadrantes de $25 \mathrm{~m}^{2}$ foram definidos nas praças e as amostras foram colhidas de quatro pontos distintos de cada quadrante, totalizando 29 quadrantes. O número de amostras dentro do espaço amostral foi formado por um pool, sendo coletadas em número aleatório por praça, com no mínimo duas coletas por quadrante. Estes quadrantes foram representados como uma amostra.

Com o auxilio de espátulas e luvas, foram coletadas aproximadamente cinco gramas de excretas envelhecidas e secas, pelo fato de oferecem substrato favorável ao desenvolvimento da levedura e apresentarem menor quantidade de bactérias, não havendo competição nutricional entre estes microrganismos (REOLON et al., 2004). As amostras foram resgatadas em dias alternados, entre as 14 e 17 horas, em diferentes condições de temperatura e umidade, com algumas amostras que se apresentaram expostas à radiação solar, enquanto outras em condições de sombreamento. 
Após coletadas, as excretas foram acondicionadas em potes estéreis devidamente identificados e encaminhados ao Laboratório de Cultura e Microbiologia da Universidade do Planalto Catarinense (UNIPLAC) onde permaneceram armazenadas em temperatura ambiente por 48 horas. As amostras foram homogeneizadas com $2 \mathrm{~mL}$ de solução fisiológica estéril, o sobrenadante foi aspirado e semeado em placas de Petri; cada amostra foi semeada em uma placa contendo o meio de cultura de Ágar Níger, conforme indicação de Santos et al. (2009). As placas foram incubadas em estufa, por sete dias, à temperatura de 29-30 ${ }^{\circ} \mathrm{C}$ (MACHADO et al., 1993). Para confirmação do crescimento colonial em meio de cultura com Cryptococcus spp. foi observada a coloração que varia de bege claro a marrom. As colônias com características de levedura foram submetidas à prova da urease, enzima que auxilia no diagnóstico (FISHER; COOK, 2001).

A identificação macroscópica prévia levou em conta as características de crescimento de colônias compatíveis com Cryptococcus spp. que apresentaram aspecto cremoso, brilhante e úmido, de coloração branco-amarelada tornando-se bege escuro a marrom com o passar do tempo de incubação. Estas colônias foram submetidas à confecção de lâminas para a análise microscópica do fungo e coradas com tinta da China, o corante padrão para visualização da cápsula fúngica e posteriormente observadas em microscopia óptica em aumento de 1000X.

\section{RESULTADOS E DISCUSSÃO}

Das 195 amostras de excretas de pombos coletadas das seis praças amostradas, 149 (76,41\%) apresentaram crescimento de colônias de tamanho médio e textura cremosa o que levou ao diagnóstico presuntivo de Cryptococcus spp., porém a positividade para a levedura foi de 7,69\% (15), diagnosticadas através do crescimento da cultura com textura e coloração compatíveis para a levedura, prova da urease e análise microscópica.

Em relação ao diagnóstico de Cryptococcus spp. nas praças, sete amostras foram positivas para a praça João Costa, quatro obtidas no Calçadão Tulio Fiuza de Carvalho, três na Praça João Ribeiro e uma amostra na praça Jonas Ramos. 
O uso da prova da urease para o diagnóstico de Cryptococcus é devido a capacidade do fungo em hidrolisar ureia por meio de uma metaloenzima, resultando em amônia e carbamato, com alteração do pH do meio, mudando a coloração de amarelo a rosa. Esta capacidade de hidrolisar ureia é importante para diferenciar outras espécies de leveduras como Candida spp. (SIDRIM; ROCHA, 2004). O uso do meio de Níger se deve a melhor visualização e caracterização da colônia que os métodos tradicionais para a cultura fúngica. A capacidade de produção de melanina com formação de colônias marrons em meios com extrato de sementes de Niger é característica de $C$. neoformans e $C$. gattii, não ocorrendo em outras espécies do gênero Cryptococcus, nem outras leveduras de interesse médico (SANTOS et al., 2009).

A realização do estudo durante o período de inverno explica-se pela influência dos fatores ambientais no isolamento e diagnóstico da levedura, baseado em que o clima de uma determinada região aliado à temperatura, umidade e exposição à radiação solar podem influenciar na recuperação e isolamento de cepas do fungo de origem ambiental (QUINTERO et al., 2005).

Segundo a publicação técnica Consenso em Criptococose (2008), o fungo cresce melhor a temperatura de $30^{\circ} \mathrm{C}$ e não cresce a temperaturas acima de $40{ }^{\circ} \mathrm{C}$, bem como é sensível à luz solar direta. Como Lages está situada na região serrana de Santa Catarina, uma das regiões mais frias do Brasil, o fator temperatura poderia ter influência no isolamento do fungo. Durante os meses de estudo a média das temperaturas mínimas foi de 7 e $8{ }^{\circ} \mathrm{C}$ e máxima de 16 e $17{ }^{\circ} \mathrm{C}$ em 2011 e 2012, respectivamente, sendo necessário observar o comportamento do crescimento do fungo nestas condições.

Quanto ao isolamento de Cryptococcus spp. de excretas de pombos, a positividade em nosso estudo foi baixa em relação a estudos semelhantes realizados no Brasil. Na região sul, Reolon et al. (2004) detectaram o fungo em 100\% de amostras de Porto Alegre e Faria et al. (2010) em Pelotas registraram positividade de 26,9\% em amostras ambientais. Abegg et al. (2006) analisaram excretas de 59 espécies de aves de cativeiro mantidas em gaiolas em um jardim zoológico do Rio Grande do Sul, as aves eram pertencentes a 12 ordens diferentes. Trinta e oito isolados ambientais de C. neoformans foram obtidos apenas a partir de 
Psitacídeos (Psittacidae, Cacatuidae e Psittacula), 33 isolados (87\%) foram da var. grubii, o sorotipo A, tipo VNI e cinco (13\%) foram C. gattii. Na região centro-oeste, em Campo Grande (MS), Filiú et al. (2002) diagnosticaram C. neoformans em 50\% das 20 amostras de excretas de aves de cativeiro; em Goiânia, Kobayashi et al. (2005) isolaram a levedura em 20,3\% de excretas de pombos. Na região sudeste, Passoni et al (1998), analisaram 824 amostras, incluindo poeira domicilar, solo e excretas de aves no Rio de Janeiro com C. neoformans sendo isolado em 20 (13\%) das moradias. Foi observado que entre as casas com a presença do fungo havia pacientes HIV com criptococose. Soares et al. (2005) obtiveram a positividade de $13,9 \%$ de C. neoformans em Santos (SP); Baroni et al. (2006) encontraram 37,8\% da levedura em excretas de pombos no Rio de Janeiro; Contin et al. (2011) em Caratinga (MG) identificaram a levedura em 27 (90\%) amostras de 10 locais pesquisados. Tortora et al. (2010) confirmam a ampla distribuição de Cryptococcus spp. no solo, onde há contaminação por fezes de pombos, como também em poleiros e ninhos de pombos e nas janelas de edifícios urbanos.

A presença do fungo em amostras ambientais é maior quando estas se encontram protegidas das condições meteorológicas (KOBAYASHI et al., 2005). Entretanto, valores menores de ocorrência foram obtidos, recentemente, por Takahara et al. (2013), que diagnosticaram $C$. neoformans em 6,6\% de amostras ambientais de 49 locais, entre públicos e particulares, da cidade de Cuiabá (MT) e Yamamura et al. (2013) com 0,84\% de positividade na cidade de Londrina (PR).

A diferença entre os resultados de isolados de Cryptococcus spp. pode estar relacionada aos locais e período de coleta, embora não se possa afirmar se em dias de frio intenso o comportamento do microrganismo seria o mesmo que em dias quentes. Não se pode determinar se as características climatológicas da região de Lages apresentam as condições ideais para a propagação do fungo, em virtude de ter médias de temperaturas anuais mais baixas que de outras regiões, além da umidade e limpeza do ambiente.

A baixa ocorrência no isolamento do Cryptococcus spp. neste estudo não isenta do risco para a saúde humana, visto que os pombos fazem parte da paisagem dos centros urbanos como ocorre na cidade de Lages. A multiplicação do fungo em fezes e ambientes com falta de 
limpeza aliados ao aumento da temperatura, propicia aos basidiósporos do fungo, por via aerógena a partir dos excrementos, a contaminação de Passeriformes e Psitaciformes (LUGARINI et al., 2008), reservatórios da levedura, bem como a contaminação de outros ambientes nas proximidades dos nichos de pombos (ROSÁRIO et al., 2008).

Na maioria das praças centrais de Lages ocorre a limpeza diária do ambiente, o que impede o acúmulo de fezes. A praça João Costa e o calçadão Tulio Fiuza de Carvalho apresentaram maior número de amostras positivas, com relação direta ao maior número de pombos, provavelmente como consequência do maior fluxo de pessoas que alimentam as aves. As praças que tiveram isenção ou menor contaminação por Cryptococcus spp. apresentam circulação mais restrita de pessoas e reduzida população de pombos, pela reduzida oferta de alimento. Para Faria et al. (2010), excluindo-se as características ambientais, a quantidade de excretas pode interferir nos resultados, pois a baixa quantidade pode dificultar a semeadura e isolamento, fator que muitas vezes foi um obstáculo neste trabalho, devido a limpeza dos locais de coleta com pouco material depositado no ambiente. Outro fator que pode ter contribuído para a baixa contaminação é a presença de excretas úmidas, que pode ter ocorrido em decorrência do alto índice pluviométrico registrado durante o período do inverno de 2012, além da limpeza diária executada. A umidade dos excrementos interfere na sobrevivência do fungo, pois promove maior quantidade e atividade das bactérias causando a alcalinização do substrato e a competição alimentar com $C$. neoformans inibindo o crescimento da levedura (FISHER;COOK, 2001).

A população de pombos vem se tornando um problema ambiental e de saúde pública, devido a preferência por abrigos em locais de difícil acesso como torres de igreja e edifícios, nesses nichos as formas evolutivas do fungo, podem permanecer por anos (CERMEÑO, 2006; ROSARIO et al., 2008; TAKAHARA et al., 2013). Ainda não foi esclarecido o motivo da presença do fungo nos excrementos de pombos, uma vez que não é isolado diretamente do trato intestinal destas aves (REOLON et al., 2004). O principal mecanismo de infecção pelo fungo é através da inalação ou ingestão de partículas do microrganismo, onde a levedura assume caráter saprofítico em aves, porém estes animais são protegidos pela alta temperatura corporal (KWON-CHUNG; BENNETT, 1992; MALIK et al., 2003). 
A limpeza diária dos ambientes públicos, além de retirar as excretas dos pombos, pode proporcionar umidade que impede o desenvolvimento do fungo, sendo uma medida profilática que pode ser adotada em cidades onde esta prática pode ser realizada (CIVILA; CONTI-DIAZ, 1976).

As praças são locais preferenciais para o lazer das pessoas, principalmente de crianças e idosos, portanto é importante campanhas de orientação à população sobre a criptococose; informar sobre os riscos do aumento da população dos pombos no perímetro urbano, mostrando as pessoas a importância em não alimentá-los. Ainda como medida de controle não permitir o contato de crianças, idosos e indivíduos imunocomprometidos com as excretas de pombos em áreas de lazer, uma vez que estas pessoas são mais suscetíveis à infecção por C. neoformans (SOLTANI et al., 2013).

\section{CONCLUSÃO}

A maioria das amostras positivas foram colhidas em locais com maior concentração de pombos e fluxo de pessoas. Esses resultados demonstram a necessidade de alertar a população, através de campanhas educativas, sobre os riscos de contrair a criptococose e a importância de não alimentar os pombos em áreas de lazer, a principal estratégia no controle populacional dos pombos.

\section{Cryptococcus spp. IN PIGEONS (Columba livia) DROPPINGS IN PUBLIC SQUARES OF LAGES, SANTA CATARINA}

\section{ABSTRACT}

ryptococcosis is an opportunistic fungal infection caused by the encapsulated yeast Cryptococcus mainly C. neoformans and C. gatti, acquired by the inhalation of infectious propagules from the environment. Pigeons (Columba livia) are associated with the infection, acting as natural reservoirs of the fungus, given the large number of these animals in public areas, mainly in places with high concentration of people. A total of 195 samples of pigeon droppings, collected from public squares downtown in Lages, state of Santa Catarina, Brazil, were analyzed for the isolation and diagnosis of $C$. neoformans. From 
the analyzed samples, $7.69 \%$ (15) were positive for the encapsulated yeast. Therefore, it is necessary to warn the population about the risk of cryptococcosis and discourage feeding pigeons in leisure areas, which seems to be the major control strategy.

Keywords: Cryptococcus neoformans. Pigeons. Zoonosis. Yeast. Public Square.

\section{Cryptococcus spp. EN LOS EXCREMENTOS DE PALOMAS (Columba livia) DE ESPACIOS PÚBLICOS DE LAGES, SANTA CATARINA}

\section{RESUMEN}

a criptococosis es una infección micótica común oportunista causada por la levadura encapsulada del genero Cryptococcus, principalmente $C$. neoformans y Cryptococcus gattii. El hongo es adquirido por la inhalación de material de origen ambiental y más raramente a través de trasplantes de órganos en individuos inmunodeprimidos. La paloma doméstica (Columba livia) esta relacionada con la infección, especialmente como reservorios naturales del hongo y debido a la alta concentración de estos animales en ambientes públicos, especialmente en lugares de gran movimiento de personas. Para el aislamiento y el diagnóstico de $C$. neoformans, 195 muestras fueron analizadas de excrementos de palomas de seis plazas ubicadas en la región central de la ciudad de Lages, SC. De las muestras analizadas $7,69 \%$ (15) fueron positivas para levadura encapsulada. Basado en que la positividad ocurrió en sitios con mayor concentración de palomas y mayor flujo de personas es necesario alertar al público sobre el riesgo de contraer la criptococosis y de la necesidad de que las mismas no alimenten a las palomas en las áreas públicas. Esta sería la principal estrategia para controlar la población de las mismas.

Palabras clave: Cryptococcus neoformans. Levadura. Palomas. Zoonosis. Plaza pública.

\section{REFERÊNCIAS}

ABEGG, M. A.; FAGANELLO, J.; VALENTE, P.; et al. Cryptococcus neoformans and Cryptococcus gattii isolated from the excreta of Psittaciformes in a Southern Brazilian Zoological Garden. Mycopathologia, v. 161, n. 2, p. 83-91, 2006.

BARONI, F. A.; PAULA, C. R.; SILVA, E. G.; et al. Crytococcus neoformans strains isolated from church towers in Rio de Janeiro city, RJ, Brazil. Revista do Instituto de Medicina Tropical, v. 48, n. 2, p. 71-5, 2006.

CERMEÑO, J. R.; HERNÁNDEZ, I.; CABELLO, I.; et al. Cryptococcus neoformans and Histoplasma capsulatum in dove's (Columbia livia) excreta in Bolívar State, Venezuela.

Revista Latinoamericana de Microbiologia, v. 48, n. 1, p. 6-9, 2006. 
CIVILA, E.; CONTI-DIAZ, I. A. Aislamiento de Cryptococcus neoformans de excretas secas de palomas en la ciudad de Montevideo. Revista Uruguaya de Patologia Clinica e Microbiologia, v. 3, p. 41-48, 1976.

CONSENSO EM CRIPTOCOCOSE. Relatório Técnico. 2008. Revista da Sociedade Brasileira de Medicina Tropical, v. 41, n. 5, p. 524-544, 2008.

CONTIN, J. T.; QUARESMA, G. S.; SILVA, E. F.; et al. Ocorrência de Cryptococcus neoformans em fezes de pombos na cidade de Caratinga, MG-Brasil. Revista de Medicina de Minas Gerais, v. 21, n. 1, p. 19-24, 2011.

ENGELTHALER, D.M.; HICKS, N.D.; GILLECE, J. D.; et al. Cryptococcus gattii in North American Pacific Northwest: Whole-Population Genome Analysis Provides Insights into Species Evolution and Dispersal. mBio, v. 5, n. 4. 2014.

FARIA, R. O.; NASCENTE, P. S.; MEINERZ, A. R. M.; et al. Ocorrência de Cryptococcus neoformans em excretas de pombos na Cidade de Pelotas, Estado do Rio Grande do Sul. Revista Brasileira de Medicina Tropical, v. 43, n. 2, p. 198-200, 2010.

FILIÚ, W. F.; WANKE, B.; AGUENA, S. M.; et al. Cativeiro de aves como fonte de Cryptococcus neoformans na cidade de Campo Grande, Mato Grosso do Sul, Brasil. Revista da Sociedade Brasileira de Medicina Tropical, v. 6, n. 35, p. 591-5, 2002.

FISHER, F.; COOK, N. B. Micologia: fundamentos e diagnóstico. Rio de Janeiro: Revinter, 2001. 337p.

GRANADOS, D. P.; CASTAÑEDA, E. Isolation and characterization of Cryptococcus neoformans varieties recovered from natural sources in Bogotá, Colombia, and study of ecological conditions in the area. Microbial Ecology, v. 49, n. 2, p. 282-90, 2005.

HUFFNAGLE, G. B .A.; CASADEVALL, A.; PERFECT, J. R. Cryptococcus neoformans. Mycophatologia, v. 147, n. 1, p. 59-60, 1999.

IKEDA, R.; SHINODA, T.; FUKAZAWA, Y.; et al. Antigenic characterization of Cryptococcus neoformans serotypes and its application to serotyping of clinical isolates. Journal of Clinical Microbiology, v. 16, n. 1, p. 22-9, 1982.

KOBAYASHI, C. C. B. A.; SOUZA, L. K. H.; FERNANDES, O. F. L.; et al. Characterization of Cryptococcus neoformans isolated from urban environmental sources in Goiânia, Goiás State, Brazil. Revista do Instituto de Medicina Tropical, v. 47, n. 4, p. 203-207, 2005.

KON, A. S. Consenso em criptococose. Revista da Sociedade Brasileira de Medicina Tropical, v. 41, n. 5, p. 524-544, 2008. 
KWON-CHUNG, K. J.; BENNETT, J. E.; Epidemiologic differences between the two varieties of Cryptococcus neoformans. American Journal of Epidemiology, v. 120, n. 1, p. 123-130, 1984.

KWON-CHUNG, K. J.; BENNETT, J. E. Medical Mycology. Philadelphia: Lea \& Figiber, 1992, $866 p$.

LIN, X.; HEITMAN, J. The biology of the Cryptococcus neoforman species complex. Annual Review of Microbiology, v. 14, n. 5, p. 69-105, 2006.

LUGARINI, C.; GOEBEL, C. S.; CONDAS, L. A.; et al. Cryptococcus neoformans isolated from Passerine and Psittacine Bird excreta in the state of Paraná, Brazil. Mycopathologia, v. 166, n. 2, p. 61-69, 2008.

MACHADO, C. C.; AMARAL, A. A.; SEVERO, L. C. Cryptococcus neoformans var. neoformans isolado do solo. Revista do Instituto de Medicina Tropical, v. 35, n. 1, p. 77-79, 1993.

MALIK, R.; KROCKENBERGER, M. B.; CROSS, G.; et al. Avian cryptococcosis. Medical Mycology, v. 41, n. 2, p. 115-124, 2003.

MEYER, W.; TRILLES, L. Genotyping of the Cryptococcus neoformans/C. gattii species complex. Australian Biochemist, v. 41, n. 1, p. 11-15, 2010.

PASQUAlOtTO, A. C.; SEVERO C. B., OliVEIRA, F. M.; SEVERO, L. C. Cryptococcemia. An analysis of 28 cases with emphasis on the clinical outcome and its etiologic agent. Revista Iberoamericana de Micologia, v. 21, n. 3, p. 143-6, 2004.

PASSONI, L. F. C.; WANKE, B.; NISHIKAWA, M. M.; et al. Cryptococcus neoformans isolated from human dwellings in Rio de Janeiro, Brazil: An analysis of domestic environment of AIDS patients with and without cryptococcosis. Medical Mycology, v. 36, n. 5, p. 305-11, 1998.

QUEIROZ, J. P. A. F.; SOUSA, F. D. N.; LAGE, R. A.; et al. Criptococose - Uma Revisão Bibliográfica. Acta Veterinária Brasilica, v. 2, n. 2, p. 32-38, 2008.

QUINTERO, E.; CASTAÑEDA, E.; RUIZ, A. Environmetal distribution of Cryptococcus neoformans in the department of Cundinamarca - Colombia. Revista Iberoamericana de Micologia, v. 22, n. 2, p. 93-98, 2005.

REOLON, A.; PEREZ, L. R. R.; MEZZARI, A. Prevalência de Cryptococcus neoformans nos pombos urbanos da cidade de Porto Alegre, Rio Grande do Sul. Jornal Brasileiro de Patologia e Medicina Laboratorial, v. 40, n. 5, p. 293-8, 2004.

ROSARIO, I.; ACOSTA, B.; COLOM, F. La Paloma y otras aves como reservorio de Cryptococcus spp. Revista Iberoamericana de Micologia, n. 25, p. S13-S18, 2008. 
SANTOS, L. L.; FERREIRA, F. M.; LOPES, S. F.; et al. Pesquisa de Cryptococcus neoformans e Candida spp. em excretas de Psitacídeos e passeriformes cativos. Arquivos de Ciências Veterinárias e Zoologia, v. 12, n. 1, p. 5-9, 2009.

SEVERO, C. B.; GAZZONI, A. F.; SEVERO, L. C. Curso de Atualização - Micoses: Capítulo 3 Criptococose pulmonar. Jornal Brasileiro de Pneumologia, v. 35, n. 11, p. 1136-1144, 2009.

SIDRIM, J. J. C.; ROCHA, M. F. G. Micologia médica à luz de autores contemporâneos. Rio de Janeiro: Guanabara Koogan, 2004, 388p.

SOARES, M. C. B.; PAULA, C. R.; DIAS, A. L. T.; et al. Environmental strains of Cryptococcus neoformans variety grubii in the city of Santos, SP, Brazil. Revista do Instituto de Medicina Tropical de São Paulo, v. 47, n. 1, p. 31-36, 2005.

SOLTANI, M.; BAYAT, M.; HASHEMI, S. J.; et al. Isolation of Cryptococcus neoformans and other opportunistic fungi from pigeon droppings. Journal Research in Medical Sciences, $v$. 18, n. 1, p. 56-60, 2013.

TAKAHARA, D. T.; LAZÉRA, M. S.; WANKE, B.; et al. Primeiro registro de Cryptococcus neoformans em excretas de pombos provenientes de locais públicos e residenciais de área metropolitana de Cuiabá, Estado do Mato Grosso, Brasil. Revista do Instituto de Medicina Tropical, v. 55, n. 6, p. 371-376, 2013.

TORTORA, G. J., FUNKE, B. R., CASE, C. L. Microbiologia. Porto Alegre: Artmed. 2010. 894p.

YAMAMURA, A. A. M.; FREIRE, R. L.; YAMAMURA, M. H.; et al. Estudo dos nichos ecológicos de leveduras patogênicas das espécies Cryptococcus neoformans e Cryptococcus gatti na cidade de Londrina, PR. Sêmina: Ciências Agrárias, v. 34, n. 2, p. 793-804, 2013.

Autor para correspondência: Sandra M. T. Marques Faculdade de Veterinária, Av. Bento Gonçalves, 9090, Bairro Agronomia-Cx. P. 91.540-000, Porto Alegre, RS. smtmuni@hotmail.com 